\title{
Young Massive Clusters - Formation Efficiencies and (Initial) Mass Functions
}

\author{
Søren S. Larsen ${ }^{1}$ \\ Astronomical Institute, Utrecht University, Princetonplein 5, NL-3584 CC, The \\ Netherlands. larsen@astro.uu.nl
}

\begin{abstract}
Summary. Globular clusters are often assumed to be good tracers of major star formation episodes in their host galaxies. While observations over the past 2 decades have confirmed the presence of young objects with globular cluster-like properties in many galaxies, it is still not well understood exactly how the formation efficiency of bound star clusters relative to field stars and their mass spectrum depend on external factors. The cluster initial mass function typically appears to be consistent with a power-law with a slope $\sim-2$, but most attempts to constrain any upper limit on the CIMF have been limited by size-of-sample effects. However, evidence is starting to accumulate for possible truncation of the cluster mass function. It is tentatively suggested that the upper mass limit may currently be at $\sim 10^{5} \mathrm{M}_{\odot}$ in the Milky Way disk, while there are indications that it is $\sim 5 \times 10^{5} \mathrm{M}_{\odot}$ in M51 and about $2 \times 10^{6} \mathrm{M}_{\odot}$ in the Antennae. Some extreme starbursts (e.g. Arp 220, NGC 7252) are (or were) able to form clusters as massive as $\sim 10^{7} \mathrm{M}_{\odot}$. The overall formation efficiency of star clusters (relative to field stars) in the Galactic bulge may not have been much different from that in the disk today, but was probably significantly higher for metal-poor GCs in halos.
\end{abstract}

\section{YMCs - Guides to Young Stellar Populations?}

Three or four decades ago, globular cluster (GC) formation was thought by many to be a phenomenon occurring only in the early Universe (e.g. 20]). In the meantime, young, compact star clusters with masses in the range $10^{4}$ $10^{6} \mathrm{M}_{\odot}$ have been found in many different galaxies (e.g. 18, 21, 24]), and there is a growing concensus that these "Young Massive Clusters" (YMCs) may well be young analogues of the old GCs associated almost universally with the spheroidal components of galaxies. By implication, GCs have become potentially interesting not just as fossil left-overs from the early Universe, but more generally as test particles for studies of extragalactic stellar populations.

In keeping with this spirit, contemporary observing proposals or papers on GCs often include an introductory remark along the lines of: "GCs are thought to be good tracers of the major star forming episodes in their host galaxies". YMCs/GCs may indeed trace star formation quite generally, but it is also clear that some caution must be exercised. For example, the number of GCs per field star varies from galaxy to galaxy (the classical "specific frequency problem"), as well as between stellar populations within galaxies 14, 11. This 
must be due to differences in the formation efficiency of GCs relative to field stars, in the GC survival rates, or (more likely) some combination of the two. Of the roughly 150 catalogued GCs in our own Galaxy [13, about 2/3 are associated chemically, spatially and kinematically with the halo, while this is true for only $\sim 1 \%$ of the stars. Conversely, some $90 \%$ of the stellar mass resides in the thin disk of our Galaxy, while no GCs are currently known to be associated with this component. Even though some quite massive clusters might be located in remote parts of the Galactic disk [7] 10, a simple scaling by stellar mass of the GCs in the bulge or halo would predict hundreds or thousands of GCs in the disk, seemingly at odds with the observations.

All this is of course well known, and is the reason why GCs are often assumed to trace only "major star forming episodes". The problem then remains to define when a star forming episode qualifies as "major". Perhaps GCs mainly trace spheroid formation [4, but some stars currently residing in spheroids may originally have formed in disks. As an example, it is illustrative to consider the outcome of merging two Milky Way galaxies: The merger product would contain about 200 metal-poor and 100 metal-rich pre-existing GCs from the progenitor galaxies, assuming no GCs are destroyed. The current gas mass in the Milky Way is about $0.5 \times 10^{10} \mathrm{M}_{\odot}[6$, which is about half the mass of the bulge. Assuming that the merger would form GCs with the same efficiency as the bulge, using all the available gas, about 50 new metalrich GCs would form (and survive). The resulting GC population would then consist of three major sub-components: metal-poor, old GCs originating in the progenitor galaxy halos, moderately metal-rich GCs from the pre-existing bulges, and very metal-rich GCs formed in the merger. The estimate of the number of new GCs is obviously very crude. However, the main point here is that while the majority of the stars in the resulting spheroid (about 90\%) would have formed in the disks already before the merger took place, their formation history might not be reflected in the GC system.

Mergers at higher $z$ were likely more gas-rich, and the discrepancy between the star- and GC formation histories may have been less extreme than in the example outlined above. Nevertheless, since GCs play such an important role in attempts to constrain the star formation histories of early-type galaxies, there is a clear need to also quantify the limitations better.

\section{What can we learn from studies of YMCs?}

In order to understand the differences between properties of star cluster populations in different galaxies better, it is useful to divide the problem into three sub-problems which can, to a large extent, be addressed separately: 1) Understanding the cluster (initial) mass function (CIMF) - Is it universal, or do some parameters (e.g. the slope or upper mass limit) vary as a function of external parameters (star formation rate, gas pressure/density)? 2) The cluster formation efficiency relative to "field" stars: Again, how does this depend 
on external factors? 3) Disruption effects: these are driven both by internal mechanisms (two-body relaxation, binaries, black holes) and external factors (shocks, interactions with giant molecular clouds, tidal fields). However, it is a hard problem and progress has been slow in making reliable, quantitative predictions for the time scales involved, at least until very recently.

In the following I will concentrate mainly on the first item in the list, with only a few remarks about formation efficiencies. Disruption effects are covered elsewhere in this volume (e.g. Baumgardt, De Marchi, Vesperini).

\subsection{The Cluster Initial Mass Function}

The number of galaxies with reliable constraints on the CIMF remains small. Probably the best-known example is the Antennae, where the CIMF appears well approximated by a power-law $d N / d M \propto M^{\alpha}$ with $\alpha \approx-2$ over the range $10^{4}<M / M_{\odot}<10^{6}[27$. Similar mass functions have been found in M51 3], NGC 3310 and NGC 6745 [8], the Milky Way [9] and in the LMC [15, although not all studies cover the same mass range. It seems reasonable to conclude that star clusters typically form with a mass spectrum that can be well approximated by a uniform power-law over some mass range. It should be mentioned that some dwarf galaxies contain a few clusters which are much brighter than one would expect from the total number of star clusters in those galaxies 2. Here, however, I mainly focus on the opposite problem, i.e. whether there might be a truncation of the CIMF at some upper mass $M_{\text {trunc }}$, thus inhibiting efficient formation of clusters above a certain mass limit ( $\left.M_{\text {trunc }}\right)$ under certain circumstances (e.g. in the Milky Way disk today).

\subsection{Some considerations on the Milky Way}

Starting again with the Milky Way, it is interesting to consider the consequences of postulating that young clusters are drawn purely at random from a power-law distribution with $\alpha=-2$. The current star formation rate in bound star clusters in the solar neighbourhood is estimated to be around $5.2 \times 10^{-10} \mathrm{M}_{\odot} \mathrm{yr}^{-1} \mathrm{pc}^{-2}[16$. Assuming for simplicity a constant cluster formation rate in the Galactic disk over the past 10 Gyrs and that the cluster formation rate in the Solar neighbourhood is representative for the disk as a whole, this corresponds to $\sim 10^{9} \mathrm{M}_{\odot}$ formed in bound clusters within the Solar circle $(8.5 \mathrm{kpc})$. This is most likely a conservative estimate and the true number may well be significantly higher. Sampling these clusters from a power-law CIMF for $10^{2}<M / M_{\odot}<10^{7}$, one predicts a total of close to $9 \times 10^{5}$ clusters formed over the lifetime of the disk, of which 9000,900 and 80 have $M>10^{4} M_{\odot}, M>10^{5} M_{\odot}$ and $M>10^{6} M_{\odot}$. These numbers do not depend strongly on the adopted upper and lower integration limits, although they do depend on the CIMF slope - a steeper slope implies a more bottom-heavy CIMF, with fewer high-mass clusters. 
There are two consistency checks worth making: first, the stellar mass of the Milky Way bulge is about $10 \%$ of that of the disk. If clusters formed with the same efficiency in the bulge as they do in the disk now, one might expect about 90 clusters with $M>10^{5} M_{\odot}$ in the bulge, of which 8 have $M>10^{6} M_{\odot}$. The actual observed numbers of GCs in the bulge are smaller by a factor of 4 , which may be partly due to disruption effects. However, there is no indication that clusters formed with a higher efficiency in the bulge, and the number of GCs observed in the bulge may be roughly consistent with a formation efficiency (relative to field stars) similar to that observed today in the Galactic disk. The numbers for the halo are more difficult to reconcile with this picture, since the halo has about twice as many GCs as the bulge but an order of magnitude fewer stars. This suggests a higher formation efficiency of metal-poor halo GCs, consistent with observations of early-type galaxies [14, 11]. The distinction between halo (metal-poor) GCs and all other star clusters may be more fundamental than the one between old GCs in general and present-day star formation. Note that these arguments are slightly different from those of McLaughlin [19] who argued for a universal cluster formation efficiency relative to the total available gas mass.

Second, we can compare with the number of massive star clusters actually observed in the disk. By construction, the current formation rate agrees with the number of low-mass clusters $\left(M<10^{3} \mathrm{M}_{\odot}\right)$ observed locally, but it is interesting to see what happens when extrapolating to higher masses. Recently, at least two young clusters with masses in the range $10^{4}-10^{5} \mathrm{M}_{\odot}$ and ages $<10^{7}$ years have been identified [7] 10. If they are taken as representative of the formation rate of such objects within a distance of $5 \mathrm{kpc}$, this corresponds to $2 \mathrm{kpc}^{-2} \mathrm{Gyr}^{-1}$, or 4500 such clusters formed within the Solar circle over 10 Gyrs. Again, this is roughly consistent with the order-ofmagnitude estimates above (in fact, 8000 clusters with $10^{4}<M / M_{\odot}<10^{5}$ are predicted), suggesting that clusters like Westerlund 1 with masses up to about $10^{5} \mathrm{M}_{\odot}$ occur naturally (albeit rarely) as part of the normal hierarchy of star formation in the Milky Way disk today.

A typical disruption time for a cluster with mass $M$ in the solar neighbourhood is $t_{\mathrm{dis}}=1.3 \mathrm{Gyrs}\left(M / 10^{4} M_{\odot}\right)^{0.62}$ [16]. Assuming that this scaling remains valid for $M>10^{4} \mathrm{M}_{\odot}$, a cluster with an initial mass of $10^{5} \mathrm{M}_{\odot}$ is expected to disrupt in about 5 Gyrs while a $10^{6} \mathrm{M}_{\odot}$ cluster has a lifetime well in excess of a Hubble time. The disk should then contain about 500 clusters with masses greater than $10^{5} \mathrm{M}_{\odot}$ and still virtually all of the 80 clusters with $M>10^{6} \mathrm{M}_{\odot}$ formed over its lifetime. Of these objects, 7 should be found within a distance of $1 \mathrm{kpc}$. This estimate makes the crude assumption that clusters disrupt instantaneously, while in practice mass is lost at a nearly constant rate over the lifetime of the cluster. Although current catalogs of Milky Way open clusters are highly incomplete beyond $1 \mathrm{kpc}$, it seems unlikely that a large population of clusters with masses in the range $10^{5}-10^{6} \mathrm{M}_{\odot}$ could have been missed. It appears plausible that the CIMF 
in the Milky Way is truncated somewhere in the vicinity of $10^{5} \mathrm{M}_{\odot}$, or at least becomes much steeper than a power-law with a slope of -2 . However, it would be highly desirable to quantify better the completeness of current cluster surveys in the disk out to large distances (several kpc).

\subsection{Constraints on the CIMF in other galaxies}

Studies of the CIMF in external galaxies are complicated by the rapid change in mass-to-light ratio that characterizes simple stellar populations. Observed luminosities cannot be converted to masses unless the age of each individual cluster is known with some accuracy, which generally requires U-band imaging. Several studies have shown that the luminosity function of young star clusters generally appears to be sampled all the way up to its statistical upper limit 24, 2, 17. Even the scatter around the predicted relation is consistent with random sampling [17. No direct evidence for truncation of the LF has been found so far, suggesting that most large galaxies are physically able to form star clusters with masses at least up to about $10^{5} \mathrm{M}_{\odot}$.
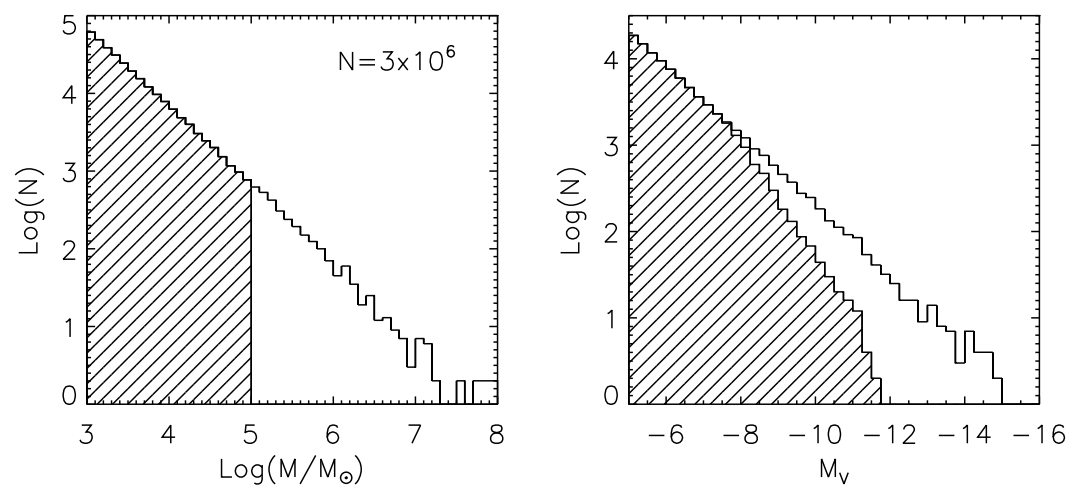

Fig. 1. Mass and $M_{V}$ distribution for a simulated cluster population with $3 \times$ $10^{6}$ clusters and $6<\log ($ age $/ y r)<9$. Hashed and outlined histograms are for mass distributions truncated at $10^{5} \mathrm{M}_{\odot}$ and $10^{8} \mathrm{M}_{\odot}$.

In general, there is no straight-forward way to infer the mass function of a cluster sample from the observed luminosity function 27. Only in the special case where the MF is a simple, untruncated power-law, or the age distribution is a delta function, will the LF and MF have the same shape. This point is illustrated in Fig. 1 which compares the mass- and luminosity functions for simulated cluster samples with power-law mass functions truncated at $10^{8} \mathrm{M}_{\odot}$ and $10^{5} \mathrm{M}_{\odot}$. The clusters were assigned random ages uniformly distributed between $10^{6}$ years and $10^{9}$ years and masses were converted to $M_{V}$ magnitudes using SSP models [5]. Mass loss and cluster disruption were 
ignored. In the left-hand panel, the MF truncation occurs as a simple cutoff, while in the LF (right) the mass cut results in a steepening of the slope at the bright end rather than any distinct cut-off in luminosity. For a real cluster population, the maximum mass may be inferred from the "bend" that occurs at $M_{V} \approx-8$ in Fig. 1 assuming that the MF is a power-law up to $M_{\text {trunc }}$ 12. Such a bend has been observed in the Antennae and M51, where it may be explained by a MF truncated near $2 \times 10^{6} \mathrm{M}_{\odot}$ and $\sim 5 \times 10^{5} \mathrm{M}_{\odot}$ 27. 12. However, in still more active galaxies such as Arp 220, NGC 7252 and NGC 1316, there are clusters with masses as high as $\sim 10^{7} \mathrm{M}_{\odot}$ [1, 26.

Let us now consider the behaviour of the following quantities as a function of the total number of clusters $(N)$ in a population with a truncation at an upper mass limit $\left.M_{\text {trunc }}: 1\right)$ the maximum mass $M_{\max }$ occurring in the population, 2) the mass of the brightest cluster $M_{\text {brightest }}$, and 3) the magnitude of the brightest cluster, $M_{V}^{\text {brightest }}$. If $N$ clusters are sampled at random from a power-law $d N / d M \propto M^{-2}$ with lower mass limit $M_{\text {min }}$, then statistically the most massive cluster will have $M_{\max }=N M_{\min }$ 24, 2. From Monte-Carlo simulations of various cluster formation histories, Weidner et al. 22] found that when clusters are sampled at random from a power-law MF, the most massive cluster is also the brightest in about $95 \%$ of the cases. If the mass function has a real physical upper limit, this is not necessarily the case.

The left panel in Fig. 22 shows the results of Monte-Carlo simulations for $M_{\text {max }}$ and $M_{\text {brightest }}$ in cluster samples with various $M_{\text {trunc }}$ limits. Clusters were drawn at random from the same population used in Fig. 1 truncated at $M_{\text {trunc }}$ values between $10^{4} \mathrm{M}_{\odot}$ and $10^{7} \mathrm{M}_{\odot}$. The median values of $M_{\max }$ and $M_{\text {brightest }}$ in 1000 experiments are shown with solid and dashed lines as a function of the number of clusters sampled. The relation predicted by pure sampling statistics is indicated as a dotted line. For small $N$ and large $M_{\text {trunc }}$, the truncation of the MF is not "felt" and the $M_{\max }$ and $M_{\text {brightest }}$ vs. $N$ relations approach the curve for random sampling, i.e. the Weidner et al. result is reproduced. Conversely, for small $M_{\text {trunc }}$ and large $N$, statistical effects become unimportant and $M_{\text {brightest }} \sim M_{\max } \sim M_{\text {trunc }}$. In between these extremes there is a regime where the mass function is likely to be sampled up to higher masses than the luminosity function, or $M_{\max }>M_{\text {brightest }}$. This turns out to be the situation encountered in many real galaxies.

For both $M_{\max }$ and $M_{\text {brightest }}$, Fig. 2 shows a fairly rapid transition between the regimes where size-of-sample effects and truncation dominate. Thus, it might seem that the observed strong relation between total number of clusters in galaxies and the luminosity of the brightest cluster [24, 2, 17] is incompatible with truncation of the CIMF playing any important role. However, as shown in the right-hand panel of Fig. 2] $M_{V}^{\text {brightest }}$ has a steep dependency on $N$ over a much wider range in $N$ than $M_{\max }$ and $M_{\text {brightest }}$. This is because the mean age of the brightest cluster shifts towards younger ages for higher $N$, as it becomes increasingly likely to encounter a cluster with mass near $M_{\text {trunc }}$ in the brief phase where the M/L ratio is very low. 

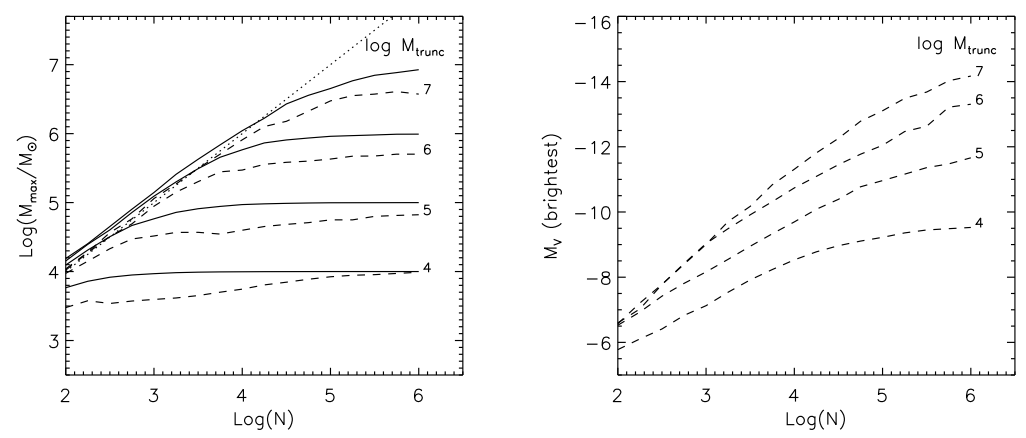

Fig. 2. Left: Mass of the most massive (solid lines) and most luminous (dashed lines) cluster as a function of total number of clusters in simulated cluster samples with various fixed upper mass limits. The dotted line is the maximum cluster mass expected from random sampling from an untruncated power-law. Right: The luminosity of the brightest cluster as a function of $N$ and $M_{\text {trunc }}$.

From the preceding discussion it should be clear that the LF can be dominated by sampling effects, even if the MF does have a physical truncation. For example, for $M_{\text {trunc }}=10^{5} \mathrm{M}_{\odot}$, the $M_{\text {max }}$ curve starts to flatten at $N \sim 10^{3}$, implying that the MF would be sampled up to its physical upper limit already in a relatively cluster-poor galaxy. The $M_{V}^{\text {brightest }}$ curve, on the other hand, would continue to rise beyond $N=10^{5}$, corresponding roughly to a Milky Way-sized galaxy (cf. Section 2.2).

The key to detecting a physical upper limit of the CIMF will be to cover a dynamic range large enough to study the LF in detail and detect signatures such as the "bend" in Fig. 1 Ultimately, however, inferences about the MF based on the LF remain indirect and dependent on assumptions about the shape of the MF, star formation history etc., and ideally it would be desirable to have direct information about the mass functions in several more galaxies. Several such studies are now underway, and may provide important insight into the MF in the not too distant future.

\section{Concluding remarks}

While research in extragalactic star clusters remains a very active field, we are still facing a number of important questions. The ubiquity of YMCs in external galaxies is now well established, but the apparent absence of a large population of clusters with masses in the range $10^{5}-10^{6} \mathrm{M}_{\odot}$ in the Galactic disk remains somewhat of a puzzle. This may suggest an upper limit to the CIMF near $10^{5} \mathrm{M}_{\odot}$ in the Milky Way, as noted already by van den Bergh \& Lafontaine [25], although studies of disk clusters are still hampered by our own location close to the Galactic plane. However, the recent realization 
that the Milky Way is still forming clusters with masses near $10^{5} \mathrm{M}_{\odot}$ hints that the CIMF in the Milky Way may not be very different from that in the LMC, the main difference being that the census of YMCs is more complete in the LMC. There is some evidence for truncation at a somewhat higher mass in M51 $\left(\sim 5 \times 10^{5} \mathrm{M}_{\odot}\right)$ and the Antennae $\left(\sim 2 \times 10^{6} \mathrm{M}_{\odot}\right)$, while Arp 220, NGC 1316 and NGC 7252 host clusters as massive as $\sim 10^{7} \mathrm{M}_{\odot}$. These galaxies also define a sequence of increasing star formation rate, suggesting that galaxies with higher SFRs are physically able to form more massive clusters. This may provide a hint as to why GC formation was common at high $z$, when SFRs and gas densities were generally higher.

\section{References}

1. N. Bastian, R.P. Saglia, P. Goudfrooij, et al.: A\&A, 448, 881 (2006)

2. O.H. Billet, D.A. Hunter \& B.G. Elmegreen: AJ, 123, 1454 (2002)

3. A. Bik, H.J.G.L.M. Lamers, N. Bastian et al.: A\&A, 397, 473 (2003)

4. J.P. Brodie, J. Strader: Ann. Rev. Astron. Astrophys., in press (2006)

5. G.A. Bruzual, S. Charlot: MNRAS, 344, 1000 (2003)

6. B.W. Carroll, D.A. Ostlie: An Introduction to Modern Astrophysics, AddisonWesley Publishing Company (1996)

7. J.S. Clark, I. Negueruela, P.A. Crowther, S.P. Goodwin: A\&A, 434, 949 (2005)

8. R. de Grijs, P. Anders, N. Bastian, et al.: MNRAS, 343, 1285 (2003)

9. B.G. Elmegreen \& Yu.N. Efremov: ApJ, 480, 235 (1997)

10. D.F. Figer, J. MacKenty, M. Robberto, et al.: ApJ, in press (astro-ph/0602146) (2006)

11. J.C. Forte, F. Faifer, D. Geisler: MNRAS, 357, 56 (2005)

12. M. Gieles, S.S. Larsen, N. Bastian, \& I.T. Stein: A\&A, 450, 129 (2006)

13. W.E. Harris: AJ, 112, 1487 (1996)

14. W.E. Harris, G.L.H. Harris: AJ, 123, 3108 (2002)

15. D.A. Hunter, Elmegreen, B.G., Dupuy, T.J., \& Mortonson, M.: AJ, 126, 1836 (2003)

16. H.J.G.L.M. Lamers, M. Gieles, N. Bastian, et al.: A\&A, 441, 117 (2005)

17. S.S. Larsen: AJ, 124, 1393 (2002)

18. S.S. Larsen: in: Planets to Cosmology: Essential Science in Hubble's Final Years, ed. M. Livio, STScI (2005)

19. D.E. McLaughlin: AJ, 117, 2398 (1999)

20. P.J.E. Peebles \& R.H. Dicke: ApJ, 154, 891 (1968)

21. F. Schweizer: In: "Extragalactic Star Clusters", ASP, eds. D. Geisler, E.K. Grebel, D. Minniti (2002)

22. C. Weidner, P. Kroupa, S.S. Larsen: MNRAS, 350, 1503 (2004)

23. B.C. Whitmore, Q. Zhang, C. Leitherer, et al.: AJ, 118, 1551 (1999)

24. B.C. Whitmore: In: "A Decade of HST Science", eds. M. Livio, K. Noll, M. Stiavelli, Cambridge: Cambridge University Press, p. 153 (2003)

25. S. van den Bergh \& A. Lafontaine: AJ, 89, 1822 (1984)

26. C.D. Wilson, W.E. Harris, R. Longden, N.Z. Scoville: ApJ, 641, 763 (2006)

27. Q. Zhang \& S.M. Fall: ApJ, 527, L81 (1999) 